\title{
BMJ Open Ontario Brain Injury Association Peer Support Program: a mixed methods protocol for a pilot randomised controlled trial
}

\author{
Sarah E P Munce, ${ }^{1}$ Susan Jaglal, ${ }^{2,3,4}$ Monika Kastner, ${ }^{5}$ Michelle L A Nelson, ${ }^{6}$ \\ Nancy M Salbach, ${ }^{1,2,4}$ John Shepherd, ${ }^{4}$ Shane N Sweet, ${ }^{7,8}$ Ruth Wilcock, ${ }^{9}$ \\ Carla Thoms, ${ }^{9}$ Mark T Bayley ${ }^{1,3}$
}

To cite: Munce SEP, Jaglal S, Kastner M, et al. Ontario Brain Injury Association Peer Support Program: a mixed methods protocol for a pilot randomised controlled trial. BMJ Open 2019;9:e023367. doi:10.1136/ bmjopen-2018-023367

- Prepublication history for this paper is available online. To view these files, please visit the journal online (http://dx.doi org/10.1136/bmjopen-2018023367).

Received 3 April 2018

Revised 14 November 2018 Accepted 20 November 2018

Check for updates

(C) Author(s) (or their employer(s)) 2019. Re-use permitted under CC BY-NC. No commercial re-use. See rights and permissions. Published by BMJ.

For numbered affiliations see end of article.

Correspondence to

Dr Sarah E P Munce;

sarah.munce@uhn.ca

\section{ABSTRACT}

Introduction The objective of this study is to conduct a pilot randomised controlled trial (RCT) of the Ontario Brain Injury Association (OBIA) Peer Support Program. The RCT is designed to evaluate the effectiveness and dose-response of the Peer Support Program in improving participation and mood for people with moderate-to-severe traumatic brain injury compared with a wait-list control group.

Methods and analysis The proposed research is a three-phase, mixed methods pilot RCT. Consistent with an integrated knowledge translation approach, the study design has been informed in consultation with the knowledge user (ie, OBIA). It will include an initial qualitative examination of barriers and enablers to the trial implementation (phase 1), a pilot RCT (phase 2) and conclude with a qualitative component (phase 3). A qualitative descriptive approach will be adopted for both qualitative phases of the study $(\mathrm{n}=20-25)$ and thematic analysis will be used. The 6 months phase-2 trial will be conducted with 60 participants. These participants will be randomised to one of three groups: a twice a week programme $(n=20)$, a once a week programme $(n=20)$ or the wait-list control group $(n=20)$. The feasibility of participant recruitment and retention, data collection, as well as participant adherence to the OBIA Peer Support Program will be evaluated. The primary outcome measure will be participation, as measured by the Participation Assessment with Recombined Tools-0bjective. Other proposed outcomes of interest will include mood, healthrelated quality of life and self-efficacy.

Ethics and dissemination Ethics approval will be obtained from the principal author's institution (University Health Network Research Ethics Board). The results of this study will inform the development of a larger scale RCT and will inform future iterations of the OBIA Peer Support Program including a revised programme curriculum. Trial registration number NCT03450460; Pre-results.

\section{INTRODUCTION}

The WHO estimates 10 million people per year are affected by a traumatic brain injury (TBI). ${ }^{1}$ The majority $(>70 \%)$ of people with moderate-to-severe TBI experience
Strengths and limitations of this study

- The use of an integrated knowledge translation approach, including consultation with Ontario Brain Injury Association (OBIA), will enhance the relevance of the findings.

- By interviewing stakeholders including individuals with traumatic brain injury, caregivers, mentors, OBIA staff members who are a part of the peer support programme, the enablers and barriers to the implementation of the protocol will be determined.

- The use of a multistage, intervention mixed-methods framework will enhance the findings of the study.

- While the wait-list control mirrors the usual process of joining the OBIA Peer Support Program, there is a possibility that participants in this arm of the trial might get better or worse during the 6 months of waiting, for reasons we may not be able to identify.

long-term physical, cognitive, emotional and behavioural challenges. ${ }^{23}$ Brain injury can disrupt executive and behavioural function caused by injury to the frontal and temporal lobes. Consequently, this injury can decrease engagement in activities, impair relationships and cause social isolation among this population. ${ }^{4}$ Rehabilitation programmes provided by interprofessional teams focus on restoring impairments, the ability to perform activities of daily living, relationships, work and social life. $^{5}$

While the health professionals are experts in the management of health conditions and the functional rehabilitation of people living with brain injury, individuals living with the effects of brain injury (or peers) are experts in how to live life with a brain injury. Peer support is defined as social emotional support that is provided by individuals with experiential knowledge and similar characteristics to the recipient. ${ }^{6}$ Other benefits of peer support 
include informational support and validation. ${ }^{7}$ The peer supporter or mentor is often considered a positive role model by the mentee. ${ }^{68}$

There is emerging evidence demonstrating the clinical effectiveness and cost-effectiveness of peer support services for individuals across a variety of chronic conditions and circumstances, ${ }^{6}{ }^{8-20}$ including adults with diabetes, ${ }^{11}$ survivors of suicide loss, ${ }^{12}$ individuals characterised by disadvantage (eg, individuals with substance abuse issues) ${ }^{13}$ patients with prostate cancer experiencing depressive symptoms, ${ }^{14}$ as well as smoking cessation in disadvantaged groups, ${ }^{19}$ and prisoners resident in adult prisons and children resident in Young Offender Institutions. ${ }^{20}$ In systematic reviews and meta-analyses of peer support interventions in a variety of different populations, inconsistent results are observed (eg, Dale and colleagues) ${ }^{21}$ (ie, only some outcomes demonstrate statistically significant findings). These studies conclude that further research is required to understand: how to tailor an intervention according to the needs of particular individuals, populations and settings; how to best implement the specific components of a peer support intervention; and the degree of sustainability of the intervention's effects. Descriptions of peer support programmes are heterogeneous and reliant on the type of setting in which they are provided; programmes also differ from one-on-one visits to online group discussions. Furthermore, programmes exhibit a variety of design choices with respect to the training of peer mentors, matches between the peer mentor and the recipient, frequency of contact and the timing post diagnosis/injury onset. ${ }^{22-24}$

\section{Effectiveness of peer support programmes in brain injury}

Two recent systematic reviews have been conducted on peer support for individuals with acquired brain injury undergoing rehabilitation ${ }^{4}$ and peer mentoring interventions for people with TBI. ${ }^{5}$

A systematic review by Wobma and colleagues ${ }^{4}$ found two low-quality randomised controlled trials (RCTs) and included a total of 126 individuals with TBI. ${ }^{23}{ }^{25} \mathrm{In}$ one of the RCTs, ${ }^{23} 12$ individuals with TBI living in the community were matched with peer mentors with TBI and compared with 18 individuals with TBI in a wait-list control group. Struchen and colleagues ${ }^{23}$ reported significant improvements in perceived social support, but no significant differences were found between groups on social integration, social network size and participation in meaningful activities. The authors concluded that further investigation is needed on the role of peer support/ mentorship as an intervention to impact improvements in social participation ${ }^{2}$ (ie, having an active role in one's community or society and engaging with a wider range of people to enhance the diversity of one's network) ${ }^{26}{ }^{27} \mathrm{In}$ the other trial, ${ }^{25} 47$ individuals with TBI who attended a peer support programme were compared with a control group of 49 individuals with TBI. In this trial, the peer support group demonstrated significant differences in improved behavioural control, decreased turmoil in the home, decreased alcohol use and improved coping, with improved physical quality of life compared with the control group.

In the second systematic review, conducted by Morris and colleagues, ${ }^{5}$ six studies met the inclusion criteria. The review included all study designs that used one-to-one peer mentoring. All of the included studies had been carried out in the USA between 1996 and 2012. The authors of the review found no significant increases in social activity or network size, but significant improvements were found in behavioural control, mood, coping and quality of life. The review concluded that there is insufficient support for the effectiveness of peer mentoring for individuals with TBI, and that the evidence that is available is derived from small studies, which are generally of low quality. The authors also identified that there was a lack of information on the content/'active ingredients' and the recommended 'dosage' of the programmes. From this review, it is clear that there remain important gaps that can be addressed by the proposed research including: understanding the 'active ingredients ${ }^{28} 29$ or mechanisms that are associated with improved outcomes, examining the impact of 'dosage' of interactions and examining why previous research has not found an impact on social integration/participation despite other benefits.

Grounding the evaluation of peer support programmes in theory could help broaden the understanding of the 'active ingredients'. For example, peer support may be grounded in Bandura's ${ }^{10}$ self-efficacy theory, a social cognitive theory that states that the key predictors of successful behaviour change are confidence (self-efficacy) in the ability to carry out an action and expectation that a particular goal will be achieved (outcome expectancy). Self-efficacy is seen as an early step in causal pathways of behaviour change in peer support programmes; increasing self-efficacy is a prerequisite for behaviour change. ${ }^{10}$ Specifically, the potential 'active ingredients' of peer support, such as using the mastery experience of others, role modelling, persuasion and reframing of physiological and affective states, may be the mechanisms to support participants' behaviour changes in this study (eg, improved social participation, mood and health-related quality of life (HRQoL) $).{ }^{10}$ In a spinal cord injury context, studies have shown the utility of other theories to better understand the how and why of peer support. ${ }^{30-32}$ These include (1) self-determination theory and its theoretical concepts of autonomy, competence and relatedness, ${ }^{33}$ and (2) transformational leadership, including idealised influence, inspirational motivation, individualised consideration and intellectual stimulation. ${ }^{34}$ Applying such theories in the brain injury context may also be useful.

To date, no trials on peer support programmes in individuals with TBI have been conducted in Canada, and none have sought to determine the 'active ingredients' of the programme and/or the ideal 'dosage' of peer support. One candidate peer support programme in the Canadian context is the Ontario Brain Injury Association 
(OBIA) Peer Support Program. The OBIA Peer Support Program was introduced in 2006 and is the only formal peer support programme for individuals with TBI in Ontario. It is provided by 15 brain injury associations across the province and coordinated by the OBIA. It is available at no cost to individuals with TBI and their family members. This peer support programme was modelled after the mentoring partnership programme used in the New York and New Jersey Brain Injury Associations which was modelled after the parent-to-parent programme. ${ }^{35}$ In the OBIA programme, mentors are individuals with a brain injury who help problem solve, and/or provide knowledge and guidance to a fellow person with a brain injury. Mentees are referred to as 'partners' and are the recipients of this support.

In the OBIA Peer Support Program, a peer support coordinator screens and interviews potential mentors, and then invites successful candidates to a 1-day group training session covering a variety of topics. ${ }^{35}$ Successful candidates are identified based on criteria provided by OBIA, including character traits that make up a good mentor, 'red flags' to look out for and character reference checks. Partners are also interviewed and screened by the peer support coordinator. ${ }^{35}$ The peer support coordinator serves as a resource to the mentor and partner or mentee (individuals with TBI or their caregivers) to initiate, continue and complete the partnership. To date, 1254 partnerships have been completed. The programme matches volunteer mentors and partners for a series of one-to-one interactions that focus on the discussion and resolution of problems or issues (ie, problem solving) related to one or several key topic areas including family and friends, resources, life changes or challenges, (healthcare) professionals, social/recreational activities, work/ employment/training/volunteering, the brain injury itself, emotions or feelings, and other issues. ${ }^{35}$ Mentors and partners may be matched according to sex, mechanism of injury, marital status and life circumstances (eg, having children). Once the match is established, the mentor and partner communicate with one another primarily by telephone until the problems or issues are either resolved or a referral to another service is made (ie, counselling or community support services). Most telephone calls last between 20 and 40 min (Thoms, personal communication, 2017) and are held weekly.

In 2012, a pre-post evaluation of the OBIA Peer Support Program was completed by the Ontario Neurotrauma Foundation. This evaluation focused on all participants of the OBIA Peer Support Program (ie, all types of acquired brain injury and family members of individuals with brain injuries) and not TBI survivors specifically. There were 168 preresponses and 182 postresponses from mentors and 236 preresponses and 126 postresponses from partners. Partners were asked to rate how helpful the mentor had been in nine areas (quality of life, communication skills, knowledge of acquired brain injury, support from others, knowledge of community resources, satisfaction with participation, overall mood, anxiety and frequency of anger). More than $60 \%$ said 'yes' for quality of life, knowledge about brain injury, overall mood and overall anxiety. The greatest proportion of partners $(73 \%)$ said that the mentor had helped them improve their overall mood. As part of this evaluation, a number of open-ended questions were also asked. Partners indicated that, with their weekly telephone calls with mentors, they felt less alone and that they could share their stories with someone who had gone through similar situations. This evaluation concluded that further study is needed in the: mentor-partner matching process (eg, What are the most important characteristics to match on? Which matching characteristics produce the best outcomes?); on the selected outcomes measures of the programme; and the (indirect) impact of the programme on family members. The need for a more rigorous evaluation using a RCT was also suggested. ${ }^{36}$

\section{Framework for proposed research}

The proposed study addresses the Community Rehabilitation component of the Promoting Reintegration and Participation section of the Clinical Practice Guideline for the Rehabilitation of Adults with Moderate to Severe TBI. ${ }^{37}$ This research was informed by the new Medical Research Council (MRC) framework for the design and evaluation of complex interventions to improve health. ${ }^{38}$ The framework proposes that there are several distinct phases in the process of development and evaluation of complex interventions. Progression from one phase to another may not be linear and is often an iterative process. There are four key elements of the new MRC framework: (1) development, (2) feasibility and piloting, (3) evaluation and (4) implementation. The proposed study focuses on the feasibility and piloting component. Furthermore, in keeping with the desire to understand the 'active ingredients' of this programme, the related MRC guidance on process evaluation of complex interventions ${ }^{39}$ will be used which considers implementation (ie, implementation process, what is delivered including fidelity, dose, adaptation and reach), mechanisms of impact (ie, participant responses to and interactions with the intervention, mediators and unexpected pathways and consequences) and context. This information will be gleaned from the qualitative components of the study as well as the mentor logs and monthly huddles, as described below, but a more deeper understanding of these key functions will be obtained in the context of the planned, larger trial.

\section{Objectives}

Objectives, hypotheses and significance

The overall objective of the current proposal is to conduct a pilot RCT designed to evaluate the effectiveness and dose-response of the OBIA Peer Support Program compared with a wait-list control group in the context of improving participation for people with moderate-to-severe TBI. The proposed pilot RCT will compare three groups: (1) partners receiving the programme twice a week, (2) partners receiving the programme once a week and (3) partners on a wait-list. The specific objectives, along with their specific phases are listed below. 
Phase 1: understanding the enablers and barriers of implementing the trial protocol

Specific objective

To understand the perceived enablers and barriers of implementing the peer support trial protocol, including the perceived enablers and barriers to participant recruitment, retention, adherence and data collection, of implementing the selected outcomes measures, and of participating in the trial, from the perspective of stakeholders (knowledge users). These include individuals with moderate-to-severe TBI, caregivers, OBIA Peer Support Program mentors, OBIA staff members who are a part of the Peer Support Program, health services and knowledge translation (KT) researchers with expertise in TBI and methodologists with expertise in clinical trials.

\section{Significance}

Results from phase 1 will be used to refine the research protocol (eg, ensure that there is a good match between the measure of participation and the content of the OBIA Peer Support Program), to optimise the outcomes of phase 2 , if necessary.

Phase 2: evaluating the feasibility of the trial protocol, conducting a pilot RCT

Specific objective

(a) To evaluate the feasibility of participant recruitment and retention, data collection, as well as participant adherence to the OBIA Peer Support Program. (b) To estimate effect sizes of the immediate impact of the 6-month OBIA Peer Support Program delivered either twice or once/ week compared with a wait-list control group on participation (primary outcome), mood, HRQoL and self-efficacy (secondary outcomes).

\section{Hypotheses}

(a) It is expected that adequate (ie, $\geq 75 \%$ ) recruitment, retention and adherence to the OBIA Peer Support Program will be achieved. (b) It is expected that the 6 month OBIA Peer Support Program delivered twice weekly will lead to greater mean improvement, on measures of participation, mood (ie, depressive symptoms), HRQoL and self-efficacy, than the same programme delivered once weekly or a wait-list control intervention (ie, a dose response effect will be observed). (c) It will be possible to estimate the magnitude of effect to allow the sample size calculation for a larger scale trial.

\section{Significance}

The results of the currently proposed research will support a future, larger scale RCT.

\section{Phase 3: exploring the impact and acceptability of the OBIA} Peer Support Program and the trial protocol

Specific objective

To explore the impact and acceptability of the OBIA Peer Support Program and the trial itself from the perspective of partners and mentors.

\section{Significance}

The results of phase 3 will lead to an understanding of the 'active ingredients' that are associated with improved outcomes and the impact of 'dosage' of interactions. Furthermore, the results of phase 3 will refine and improve future iterations of the OBIA Peer Support Program and the implementation of the larger RCT.

\section{METHODS AND ANALYSIS}

\section{Overview of study design}

The proposed research is a three-phase, mixed methods pilot RCT. Consistent with an integrated knowledge translation (IKT) approach $^{34}$ as described below, the study design has been developed in consultation with the knowledge user (ie, OBIA). It will include an initial qualitative component, a pilot RCT, and then conclude with a qualitative component. This research protocol is consistent with the Standard Protocol Items: Recommendations for Interventional Trials guidelines for the reporting of protocols for RCTs. Furthermore, this protocol has been registered with clinicaltrials.gov. The study will be conducted between 2018 and 2019

\section{Phase 1}

Design

A qualitative descriptive approach ${ }^{40}{ }^{41}$ will be adopted for phase 1 . Key informant interviews will be conducted with approximately 20 stakeholder participants. The results from this phase will be used to inform and adapt the protocol for phase 2, consistent with an IKT approach.

\section{Participants and recruitment}

A purposive sampling of participants from each of the different stakeholder groups will be used (ie, individuals with moderate-to-severe TBI, caregivers, OBIA Peer Support Program mentors, OBIA staff members who are a part of the peer support programme and researchers/ methodologists). Eligible partner participants, including caregiver partners, will include community-based (ie, no longer participating in a comprehensive rehabilitation programme) individuals who (1) have a moderate-to-severe TBI (Glasgow Coma Scale $\leq 12)^{42}$ or have cared for (ie, unpaid) an individual with moderate-to-severe TBI for at least 1 year, (2) are 18 years of age or older, (3) have participated in the OBIA Peer Support Program, (4) are fluent in English and (5) are able to provide informed consent/ have an available proxy who is able to provide informed consent. Individuals who are medically unstable or have active suicidal ideation will not be eligible to participate. To be eligible, mentor participants must have completed at least one partnership with the OBIA Peer Support Program. The OBIA Peer Support Program Database will be used to recruit the partners (including caregivers) and mentors. Online searches and/or the contacts of the principal investigators will be used to recruit the OBIA staff members and the researchers/methodologists. We will aim to recruit a total of 20 stakeholders for phase 1. 


\section{Methods}

Participants will take part in a one-on-one, semistructured telephone interview lasting approximately 45-60 min. Individuals from each of the stakeholder groups (ie, partners, mentors) will be interviewed separately. A subset of the interviews will be conducted by one of the principal investigators and the research coordinator. The remaining interviews will be conducted by the research coordinator alone. All interviews will be digitally recorded and transcribed verbatim for data analysis. The interview guides will consist of semistructured open-ended questions and will be pilot tested with various members of the research team with experience in qualitative methods, an individual with moderate and severe TBI, as well as an existing mentor. Probes or recursive questioning will be used during interviews to explore issues in greater depth and verify the interviewer's understanding of the information being collected. ${ }^{43}$

\section{Phase 2}

Design

Phase 2 will be a pilot RCT. Partner participants will be randomised to one of three groups: a twice a week programme $(n=20)$, a once a week programme $(n=20)$ or the wait-list control group $(n=20)$. These groups have been chosen to examine the dose-response of the intervention. The wait-list control was deemed acceptable by OBIA, as it is part of the usual process for interested programme participants to wait for an appropriate match. Interventions will last 6 months and evaluations will occur at baseline, 6 weeks, 3 months and 6 months postbaseline.

\section{Participants and recruitment}

Eligible partner participants will include community-based (ie, no longer participating in a comprehensive rehabilitation programme) individuals who (1) have a moderate-to-severe TBI (Glasgow Coma Scale of 12 or less), ${ }^{42}$ (2) are 18 years of age or older, (3) are fluent in English and (4) are able to provide informed consent/ have an available proxy who is able to provide informed consent. Individuals who (1) have previously participated in the OBIA Peer Support Program or another peer support/self-management programme, (2) are medically unstable and (3) have active suicidal ideation will be excluded. The same inclusion/exclusion criteria will be used for the mentor participants; in addition, they may include newly-trained mentors or mentors that have already had previous mentorship experience with the OBIA Peer Support Program. Partners and mentors with moderate-to-severe TBI will be recruited via an online advertisement posted on the OBIA website as well as the websites of the 15 participating brain injury associations. Mentor participants may also be recruited from the OBIA Peer Support Program Database. We will aim to recruit a total of 60 partners (ie, $n=20$ participants per group) and 20-40 mentors for phase 2.

\section{Intervention and control group}

Participants in the intervention groups will take part in the OBIA Peer Support Group receiving either twice a week (ie, two $20-40 \mathrm{~min}$ calls) or once a week (ie, one 20-40 min call) support. In keeping with an average duration of support, ${ }^{35}$ and for the purposes of the currently proposed study, the intervention duration will be 6 months. Participants in the control group will be assigned to a wait-list. They will receive the OBIA Peer Support Program after the intervention group (ie, after 6 months). This assignment does not represent a significant variation in the usual intake procedure, as the OBIA Peer Support Program already maintains a wait-list (Thoms, personal communication, 2017).

\section{Outcome measures}

For phase 2, baseline data including demographic and descriptive information (eg, age, sex, severity of injury, marital status), as well as the outcomes measures described below, will be measured prior to randomisation.

\section{Participation (primary outcome)}

The Participation Assessment with Recombined ToolsObjective (PART-O) ${ }^{44}$ is an objective measure of participation, describing functioning at the societal level. The instrument includes 24 items derived or modified from three measures commonly found in the TBI literature: Community Integration Questionnaire ${ }^{45}$ Participation Objective, Participation Subjective, ${ }^{46} 47$ and the Craig Handicap Assessment and Reporting Technique. ${ }^{47}$ The PART-O was developed to evaluate the effectiveness of interventions to improve social/societal functioning. The z-scores can be used to assess improvement in post-acute rehabilitation. As reported by Whiteneck et at $t^{48}$ on the development of the measure, the PART-O demonstrated a person separation of 2.47, person reliability of 0.86 , item spread of 4.25 logits, item separation of 11.36 and item reliability of 0.99 .

\section{Mood (secondary outcome)}

Mood (ie, depressive symptoms) will be measured using the nine-item Patient Health Questionnaire (PHQ9). ${ }^{49}$ It is a shorter version of the PHQ and contains nine items. It is a self-report measure of severity of symptoms of depression over the previous 2 weeks. Severity levels of symptoms of self-reported depression, as reflected in the PHQ-9 total scores, are generally graded as minimal (0-4), mild (5-9), moderate (10-14), moderately severe (15-19) and severe (20-27). A score of $\geq 10$ denotes clinically significant self-reported symptoms of depression and has been used previously with the individuals with TBI. $^{5051}$ A validity study of the measure indicated that PHQ-9 scores $>10$ had a sensitivity of $88 \%$ and a specificity of $88 \%$ for major depressive disorder. ${ }^{49}$ Criterion validity was also demonstrated in a study with 580 structured interviews conducted by mental health professionals. ${ }^{49}$ Results from these interviews showed that individuals who scored high $(\geq 10)$ on the PHQ-9 were between 7 and 13.6 
times more likely to be diagnosed with major depressive disorder by the mental health professional. ${ }^{49}$ Individuals scoring low $(\leq 4)$ on the PHQ-9 had a less than 1 in 25 chance of having major depressive disorder. ${ }^{49}$

\section{Health-related quality of life (secondary outcome)}

HRQoL will be measured using the HRQoL-Short Form-12 Health Survey (SF-12) ${ }^{52}$ The SF-12 is a shorter version of the SF-36. The SF-12 includes a physical component summary and a mental (or psychological) component summary, but does not provide information about each of the eight dimensions of the SF-36 (ie, physical functioning, social functioning, physical role, emotional role, mental health, vitality, bodily pain and general health). In a preliminary study of reliability and validity, ${ }^{52}$ test-retest correlations of 0.89 and 0.76 were demonstrated, and relative validity estimates were 0.43 to 0.93 (median $=0.67$ ) compared with the 36 -item measure. The lowest possible score is 0 and the highest is 100 (where 100 represents best health).$^{53}$ It has been used previously in a TBI population (eg, Haller et al). ${ }^{54}$

\section{Self-efficacy (secondary outcome)}

Self-efficacy will be measured using the TBI Self-efficacy Questionnaire. ${ }^{55}$ It contains four subscales including Social, Physical, Cognitive and Emotional, measuring self-efficacy for obtaining help and emotional support, managing physical symptoms, managing and compensating for cognitive symptoms, and managing emotional symptoms, respectively. Items are rated on a 10-point scale ( $1=$ not at all confident to $10=$ totally confident). Subscale scores are summed to a total score.

Higher scores indicate greater self-efficacy. It has demonstrated good internal consistency (Cronbach $\alpha=0.81-0.95) .{ }^{56}$ Within the brain injury sample, the total scale has demonstrated an internal reliability (Cronbach $\alpha$ ) of 0.93 with subscale reliabilities between 0.77 and 0.93 which closely approximate the values reported for the original subscales. ${ }^{57}$

Follow-up assessments for the outcome measures will occur at 6 weeks, 3 months and 6 months post baseline. The method of data collection (eg, paper-based, telephone, online) will be determined in phase 1 .

\section{Sample size, randomisation and blinding}

Since this is a feasibility study, a formal sample size calculation will not be performed. The recruitment of 20 participants in each arm of the trial is judged to be feasible and will produce a robust and useful amount of data. ${ }^{58}$ In fact, one of the specific objectives of this study is to evaluate the feasibility of participant recruitment and retention. The primary outcome measure, participation, as measured by the PART-O, ${ }^{44}$ will be used to inform the sample size calculation for the future, larger scale RCT. Participants (including partners and mentors) who meet the inclusion and exclusion criteria and have provided informed consent to take part in the trial will be randomised to either the intervention (twice/week or once/week programme) or the wait-list control groups. A web-based randomisation service with secure password protected login using random variable block-size will be used. Sequential sealed envelopes will be used to conceal the allocation assignment until the participant has been assigned the intervention. The primary investigator will generate the sequence while the study coordinator will enrol and assign the participants. Due to the nature of the intervention, blinding of the partners and mentors will not be possible. However, the processes of outcome assessment and data analysis will be blinded.

\section{Phase 3}

\section{Design}

A qualitative descriptive approach ${ }^{40} 41$ will be adopted again for phase 3 .

\section{Participants and recruitment}

Participants will include a subset of the partner and mentor participants from phase 2. Purposive sampling will be used to recruit partner participants in phase 3 and may include intervention group (ie, twice or once a week) and severity of TBI (ie, moderate or severe). All participating mentors will be approached about participating in the interviews. Recruitment of participants will cease once data saturation has been achieved which is the point when successive interviews become repetitive and no new responses or themes emerge. ${ }^{43}$ We will aim to recruit a total of 25 partners and mentors for phase 3 .

\section{Methods}

One-on-one semistructured telephone interviews will be conducted using the same methods as outlined in phase 1. Semistructured interview guides will explore the impact and acceptability of the programme/intervention and trial protocol. Specifically, participants will be asked about their experiences participating in the OBIA Peer Support Program and the trial itself, including the strengths and weaknesses of the programme as well as the enablers and barriers to participating in the programme. We will also explore mentor training with mentor participants. Specifically, whether any modifications/additions need to be made to the current training agenda which currently includes the following topics: (1) programme overview (eg, completion of mentor pretraining evaluations, understanding role of a mentor), (2) group exercise: getting to know your fellow mentors (eg, getting acquainted with each other, experiencing a situation that is similar to the first contact with a partner), (3) overview of communication skills and methods (eg, recognise enablers and barriers of communication), (4) programme administration (eg, expected contact between a mentor and partner, activity/contact logs), (5) initial/follow-up contacts and role play (eg, developing a rapport with your partner, role play with a volunteer mentor and partner), (6) mentoring scenarios (eg, applying effective mentoring skills, increasing confidence in participants' ability to 
make good decisions as mentors) and, (7) next steps and wrap up (eg, completion of post-training evaluations) ${ }^{35}$

\section{Data analysis}

\section{Phases 1 and 3}

For phases 1 and 3, analysis will be conducted using inductive thematic analysis as described by Braun and Clark. ${ }^{59}$ For phase 1 , the analysis will assess the perceived enablers and barriers to participant recruitment, retention, adherence and data collection, of implementing the selected outcomes measures, and of participating in the OBIA Peer Support Program and the trial itself. The results from this phase will be used to inform and adapt the protocol for phase 2. For phase 3, the analysis will assess participants' experiences with the intervention and the trial protocol itself (ie, the impact and acceptability of the programme itself/intervention and the trial protocol itself). Furthermore, the results from phase 3 will also lead to an understanding of the 'active ingredients' that are associated with improved outcomes and the impact of 'dosage' of interactions.

A subset of the interview transcripts will be initially coded by the research coordinator, giving full attention to all data. In addition, the principal investigator (SM) will independently code this same subset and they will meet to compare their codes. This step will allow for enhanced reflexivity and ensured rigour. A coding framework will be developed and applied by the research coordinator to the remaining transcripts. To facilitate the organisation and analysis of the qualitative data, the principal investigator's/research coordinator's reflective notes from the interviews, as well as the transcripts, will be entered into NVivo V.10. Following this, the codes will be clustered into groups or categories (ie, codes that share similar meanings) and the predominant themes will be identified. To maximise credibility and trustworthiness, additional members of the research team will meet with the research coordinator and principal investigator (SM) over several meetings to discuss the developing analysis. New themes will also be discussed. Together, the research team will explore various thematic maps until consensus is reached and theme labels are agreed on. The research coordinator will analyse the remaining data.

\section{Phase 2}

Baseline characteristics (eg, age, sex, severity of injury, marital status, etc) will be described and any differences between the three groups will be compared using means/ medians and frequencies and proportions. As this is a feasibility study, and the ability to collect data is being tested, no data imputation will be performed to account for missing data. ${ }^{60}$ The feasibility of recruitment will be based on whether 60 participants (ie, 20 participants in each arm of the trial) can be enrolled in the trial during the 6-month recruitment period. The number of individuals who provide informed consent per month will also be calculated. The feasibility of retention will be assessed by calculating the proportion of participants with complete data on each outcome measure at 6 weeks, 3 months and 6 months. To evaluate participant adherence, the proportion of twice-weekly and weekly sessions attended by the participants will be calculated. The proportion of participants who withdraw from the intervention at 6 weeks, 3 months and 6 months will be calculated along with the reason(s) for withdrawing. The feasibility threshold will be set at $>75 \%$ for recruitment, retention and adherence (to the intervention). To evaluate changes between groups, the analysis plan will focus on mean change scores and confidence intervals (ie, mixed-design analysis of variance model) for the outcome measures described above. Effect sizes will be calculated via Cohen d to reflect the impact of the OBIA Peer Support Program on participation, mood, HRQoL and self-efficacy.

\section{Data management}

All data will be deidentified. Quantitative study data will be entered, double entered and maintained on a secure password-protected database. Qualitative data will be deidentified at the time of transcription (eg, names will be replaced with pseudonyms). Audio recordings will be deleted from the audio recorder once uploaded to a secure network folder, and then permanently deleted once the transcript has been received and verified. All data will be stored for 10 years from the date of study closure and then destroyed.

\section{Adverse events}

Research staff will monitor adverse events through the diligent review of mentor logs. Additionally, monthly huddles with mentors will provide opportunities to report any adverse events. As part of the OBIA Peer Support Program, mentors are also required to report any issues to their peer support coordinator. Researchers and OBIA staff will remain in constant contact to share knowledge and assessment of any reported events.

\section{Public involvement}

This protocol was designed in consultation with OBIA, our community partner. The phase- 2 protocol will be further refined based on interviews with various public stakeholders, such as partners and mentors of the OBIA Peer Support Program (ie, phase 1).

\section{Strengths and limitations}

The use of an IKT approach, including consultation with the OBIA on the research objectives, eligibility criteria, data collection methods, outcomes of interest and need to address the issues of 'dosage' of interaction is considered a significant strength of the currently proposed study. By interviewing stakeholders including individuals with TBI, caregivers, mentors, OBIA staff members who are a part of the peer support programme, the enablers and barriers to the implementation of the protocol will be determined. Furthermore, the results of phase 1 will be used to inform the protocol for phase 2. Specifically, these results may refine the specific objectives, methods and timelines, identify additional challenges and mitigation 
strategies, as well as future research directions. Overall, this approach will enhance the implementability of the approach. The use of a multistage, intervention mixedmethods framework is also considered a key strength. Integration will occur at the methods (eg, data collection), and interpretation and reporting levels. ${ }^{61}$

The number of outcome measures proposed may represent a substantial burden for participants, particularly in individuals with moderate-to-severe TBI. To mitigate this potential challenge, in phase 1 , we will ask stakeholders about the suitability of the proposed measures and the strategies to minimise any identified burden (eg, use of different measures, use of shorter versions). Second, there may be a great deal of variation between mentors in how the OBIA Peer Support Program is delivered. To mitigate this potential risk, mentors will keep a log of all of their interactions with their partners, including the key components, and a weekly huddle will be held with all of the mentors as well as the research coordinator and the principal investigator to ensure the fidelity of the programme is being monitored on an ongoing basis. Third, recruitment of study participants may be challenging. However, the OBIA will assist us with the recruitment of both partners and mentors to ensure that we reach our planned sample sizes. We have allocated 6 months for the recruitment of 60 partners and 20-40 mentors. Discussions with the OBIA Executive Director and Provincial Peer Support Coordinator suggest this is feasible given the OBIA's previous experience with recruiting partners and mentors. We have planned incentive for participation by offering honoraria to compensate participants' time. Finally, while the wait-list control mirrors the usual process of joining the OBIA Peer Support Program, there is a possibility that participants in this arm of the trial might get better or worse during the 6 months of waiting, for reasons we may not be able to identify.

\section{ETHICS AND DISSEMINATION}

Any important protocol modifications will be made after communication with the research ethics board, registered at ClinicalTrials.gov, and will be described in the resulting publications. Informed consent will be obtained from all participants by the research coordinator prior to their participation in both the interviews and the trial. All investigators will have access to the final study data.

We will use a variety of passive and active end-of-grant KT approaches to disseminate our findings. The results of the study will inform future iterations of the OBIA Peer Support Program including a revised programme curriculum. Traditional KT will include dissemination through meetings locally and internationally and publications in peer-reviewed journals. Finally, partnerships with local clinical programmes and/or research initiatives will be made so that the results are disseminated in a timely and effective manner. Discussions with our stakeholder groups will inform the strategies to disseminate our findings. We will produce messages that are clear, simple and tailored to the needs of each stakeholder group, whether these are individuals or organisations, including their preferred modalities for receiving the information.

Despite the fact that community reintegration is considered the ultimate goal of rehabilitation in TBI, there is limited evidence in this domain. ${ }^{37} 62$ The currently proposed study addresses the Community Rehabilitation component of the Promoting Reintegration and Participation section of the Clinical Practice Guideline for the Rehabilitation of Adults with Moderate to Severe TBI. ${ }^{37}$ Furthermore, it is clear that further evaluation is required to clarify key questions, identify and harness the best elements of the existing OBIA Peer Support Program, identify the optimal candidates to provide and receive peer mentorship, the 'active ingredients' and dose of peer mentorship. The proposed pilot RCT will provide fundamental results to inform a larger scale RCT (ie, RCT of appropriate size) on the impact of peer support on the participation and mood of individuals with moderate-to-severe TBI.

\section{Author affiliations}

${ }^{1}$ Toronto Rehabilitation Institute, University Health Network, Toronto, Ontario, Canada ${ }^{2}$ Department of Physical Therapy, University of Toronto, Toronto, Ontario, Canada ${ }^{3}$ Institute of Health Policy, Management, and Evaluation, University of Toronto, Toronto, Ontario, Canada

${ }^{4}$ Rehabilitation Sciences Institute, University of Toronto, Toronto, Ontario, Canada ${ }^{5}$ North York General Hospital, Toronto, Ontario, Canada

${ }^{6}$ Bridgepoint Collaboratory, Lunenfeld-Tanenbaum Research Institute, Toronto, Ontario, Canada

${ }^{7}$ Department of Kinesiology and Physical Education, McGill University, Montreal, Quebec, Canada

${ }^{8}$ Center for Interdisciplinary Research in Rehabilitation of Greater Montreal, Toronto, Ontario, Canada

${ }^{9}$ Ontario Brain Injury Association, St. Catherines, Ontario, Canada

Contributors SEPM is the principal investigator and is responsible for the design and implementation of the study. SEPM developed the research protocol with MTB. RW and CT provided critical information and feedback on the OBIA programme and methods and procedures for the research protocol. All other authors (SJ, MK, MLAN, NMS, JS, SNS) provided important intellectual content and feedback on the research protocol. SEPM drafted this protocol manuscript with all other authors (SJ, MK, MLAN, NMS, JS, SNS, RW, CT, MTB) providing important feedback. All authors read and approved the final manuscript.

Funding This work was supported by the Ontario Neurotrauma Foundation grant number 2017-ABI-0BIA-1036.

Competing interests None declared.

Patient consent for publication Not required.

Provenance and peer review Not commissioned; externally peer reviewed.

Open access This is an open access article distributed in accordance with the Creative Commons Attribution Non Commercial (CC BY-NC 4.0) license, which permits others to distribute, remix, adapt, build upon this work non-commercially, and license their derivative works on different terms, provided the original work is properly cited, appropriate credit is given, any changes made indicated, and the use is non-commercial. See: http://creativecommons.org/licenses/by-nc/4.0/.

\section{REFERENCES}

1. Hyder AA, Wunderlich CA, Puvanachandra P, et al. The impact of traumatic brain injuries: a global perspective. NeuroRehabilitation 2007;22:341-53.

2. Andelic N, Hammergren N, Bautz-Holter E, et al. Functional outcome and health-related quality of life 10 years after moderate-to-severe traumatic brain injury. Acta Neurol Scand 2009;120:16-23. 
3. Sendroy-Terrill M, Whiteneck GG, Brooks CA. Aging with traumatic brain injury: cross-sectional follow-up of people receiving inpatient rehabilitation over more than 3 decades. Arch Phys Med Rehabil 2010:91:489-97.

4. Wobma R, Nijland RH, Ket JC, et al. Evidence for peer support in rehabilitation for individuals with acquired brain injury: A systematic review. J Rehabil Med 2016;48:837-40.

5. Morris RP, Fletcher-Smith JC, Radford KA. A systematic review of peer mentoring interventions for people with traumatic brain injury. Clin Rehabil 2017;31:1030-8.

6. Dennis CL. Peer support within a health care context: a concept analysis. Int J Nurs Stud 2003;40:321-32.

7. Veith EM, Sherman JE, Pellino TA, et al. Qualitative analysis of the peer-mentoring relationship among individuals with spinal cord injury. Rehabil Psychol 2006;51:289-98.

8. Sherman JE, DeVinney DJ, Sperling KB. Social support and adjustment after spinal cord injury: influence of past peermentoring experiences and current live-in partner. Rehabil Psychol 2004;49:140-9.

9. Ljungberg I, Kroll T, Libin A, et al. Using peer mentoring for people with spinal cord injury to enhance self-efficacy beliefs and prevent medical complications. J Clin Nurs 2011;20:351-8.

10. Bandura A. Self-efficacy: Toward a unifying theory of behavioral change. Psychol Rev 1977;84:191-215.

11. Patil SJ, Ruppar T, Koopman RJ, et al. Peer support interventions for adults with diabetes: a meta-analysis of hemoglobin A1coutcomes. Ann Fam Med 2016;14:540-51.

12. Bartone PT, Bartone JV, Violanti JM, et al. Peer support services for bereaved survivors: a systematic review. Omega 2017;1:30.

13. Sokol R, Fisher E. Peer support for the hardly reached: a systematic review. Am J Public Health 2016;106:1308.

14. Newby TA, Graff JN, Ganzini LK, et al. Interventions that may reduce depressive symptoms among prostate cancer patients: a systematic review and meta-analysis. Psychooncology 2015;24:1686-93.

15. Young JM, Lawrence W, Quick P. A unique role: the peer mentor on the spinal cord team at G.F. Strong Rehab Centre. Healthc Manage Forum 1999;12:51-3.

16. DeVinney DJ. The Effects of Peer-Mentoring on the Social Participation and Psychological Adjustment of Individuals with Spinal Cord Injury. Madison, Wisconsin: University of Wisconsin-Madison, 2000.

17. Boschen KA, Tonack M, Gargaro J. Long-term adjustment and community reintegration following spinal cord injury. Int $J$ Rehabil Res 2003;26:157-64.

18. Kelly EH. Community competence and violently acquired spinal cord injury: employment as a peer role model. Rehabil Psychol 2007;52:226-35.

19. Ford P, Clifford A, Gussy K, et al. A systematic review of peer-support programs for smoking cessation in disadvantaged groups. Int $J$ Environ Res Public Health 2013;10:5507-22.

20. Bagnall AM, South J, Hulme C, et al. A systematic review of the effectiveness and cost-effectiveness of peer education and peer support in prisons. BMC Public Health 2015;15:290.

21. Dale JR, Williams SM, Bowyer V. What is the effect of peer support on diabetes outcomes in adults? A systematic review. Diabet Med 2012;29:1361-77.

22. Hoey LM, leropoli SC, White VM, et al. Systematic review of peersupport programs for people with cancer. Patient Educ Couns 2008;70:315-37.

23. Struchen MA, Davis LC, Bogaards JA, et al. Making connections after brain injury: development and evaluation of a social peermentoring program for persons with traumatic brain injury. J Head Trauma Rehabil 2011;26:4-19.

24. Kessler D, Egan M, Kubina LA. Peer support for stroke survivors: a case study. BMC Health Serv Res 2014;14:256.

25. Hanks RA, Rapport LJ, Wertheimer J, et al. Randomized controlled trial of peer mentoring for individuals with traumatic brain injury and their significant others. Arch Phys Med Rehabil 2012;93:1297-304.

26. Webber M. Social capital and mental health. In: Tew J, ed. Social perspectives in mental health. Developing social models to understand and work with mental distress. London: Jessica Kingsley Publishers, 2005:90-111.

27. Webber M, Huxley P, Harris T. Social capital and the course of depression: six-month prospective cohort study. J Affect Disord 2011:129(1-3):149-57.

28. Hart T, Ehde DM. Defining the treatment targets and active ingredients of rehabilitation: Implications for rehabilitation psychology. Rehabil Psychol 2015;60:126-35.

29. Whyte J, Dijkers MP, Hart T, et al. Development of a theory-driven rehabilitation treatment taxonomy: conceptual issues. Arch Phys Med Rehabil 2014;95(1 Suppl):S24-32.
30. Sweet SN, Michalovic E, Latimer-Cheung AE, et al. Spinal cord injury peer mentorship: applying self-determination theory to explain quality of life and participation. Arch Phys Med Rehabil 2018:99:468-76.

31. Shaw RB, McBride CB, Casemore S, et al. Transformational mentoring: leadership behaviors of spinal cord injury peer mentors. Rehabil Psychol 2018;63:131-40.

32. Beauchamp MR, Scarlett LJ, Ruissen GR, et al. Peer mentoring of adults with spinal cord injury: a transformational leadership perspective. Disabil Rehabil 2016;38:1884-92.

33. In: Deci EL, Ryan RM, eds. Handbook of self-determination research Rochester, NY, US: University of Rochester Press, 2002.

34. Bass BM, Riggio RE. Transformational Leadership. 2nd edn. Mahwah, NJ: Erlbaum, 2006.

35. Ontario Brain Injury Association. Ontario brain injury association peer support program manual. Ontario, 2017.

36. Boschen KA, Gargaro J. Ontario brain injury association peer mentoring support program: external program evaluation funded by Ontario Neurotrauma Fdn. Toronto, Ontario: Ontario Neurotrauma Foundation, 2012

37. Institut national d'excellence en santé et en services sociaux (INESSS)-Ontario Neurotrauma Foundation (ONF). Clinical Practice Guideline for the Rehabilitation of Adults with Moderate to Severe TBI. https://braininjuryguidelines.org (Accessed 3 Apr 2018).

38. Craig P, Dieppe P, Macintyre S, et al. Developing and evaluating complex interventions: the new Medical Research Council guidance. BMJ 2008;337:a1655.

39. Moore GF, Audrey S, Barker M, et al. Process evaluation of complex interventions: Medical Research Council guidance. BMJ 2015;350:h1258.

40. Sandelowski M. Whatever happened to qualitative description? Res Nurs Health 2000;23:334-40.

41. Sandelowski M. What's in a name? Qualitative description revisited. Res Nurs Health 2010;33:77-84.

42. Teasdale G, Jennett B. Assessment of coma and impaired consciousness. A practical scale. Lancet 1974;2:81-4

43. Creswell JW, Plano Clark VL, Gutmann M, et al. Advanced mixed methods research designs. In: Tashakkori A, Teddlie C, eds. Handbook of mixed methods in social and behavioral research. Thousand Oaks: Sage, 2003:209-40.

44. Bogner J, Bellon K, Kolakowsky-Hayner SA, et al. Participation assessment with recombined tools-objective (PART-O). J Head Trauma Rehabil 2013;28:337-9.

45. Willer B, Ottenbacher KJ, Coad ML. The community integration questionnaire. A comparative examination. Am J Phys Med Rehabil 1994;73:103-11

46. Brown M, Dijkers MP, Gordon WA, et al. Participation objective, participation subjective: a measure of participation combining outsider and insider perspectives. J Head Trauma Rehabil 2004;19:519-26.

47. Whiteneck GG, Charlifue SW, Gerhart KA, et al. Quantifying handicap: a new measure of long-term rehabilitation outcomes. Arch Phys Med Rehabil 1992;73:519-26.

48. Whiteneck GG, Dijkers MP, Heinemann AW, et al. Development of the participation assessment with recombined tools-objective for use after traumatic brain injury. Arch Phys Med Rehabil 2011;92:542-51.

49. Kroenke K, Spitzer RL, Williams JB. The PHQ-9: validity of a brief depression severity measure. J Gen Intern Med 2001;16:606-13.

50. Fann JR, Bombardier CH, Dikmen S, et al. Validity of the patient health questionnaire- 9 in assessing depression following traumatic brain injury. J Head Trauma Rehabil 2005;20:501-11.

51. Hart T, Fann JR, Chervoneva I, et al. Prevalence, risk factors, and correlates of anxiety at 1 year after moderate to severe traumatic brain injury. Arch Phys Med Rehabil 2016;97:701-7.

52. Ware J, Kosinski M, Keller SD. A 12-Item short-form health survey: construction of scales and preliminary tests of reliability and validity. Med Care 1996;34:220-33.

53. Ware J, Snow K, Kosinski M. SF-36 Health survey: manual and interpretation guide. The Health Institute, New England Medical Center Hospitals. Boston, 1993.

54. Haller CS, Delhumeau C, De Pretto M, et al. Trajectory of disability and quality-of-life in non-geriatric and geriatric survivors after severe traumatic brain injury. Brain Inj 2017;31:319-28.

55. Cicerone KD, Azulay J. Perceived self-efficacy and life satisfaction after traumatic brain injury. J Head Trauma Rehabil 2007;22:257-66.

56. Brands I, Köhler S, Stapert S, et al. Influence of self-efficacy and coping on quality of life and social participation after acquired brain injury: a 1-year follow-up study. Arch Phys Med Rehabil 2014:95:2327-34. 
57. Lorig K, Stewart A, Ritter P, et al. Outcome Measures for Health Education and other Health Care Interventions. Thousand Oaks: Sage Publications, 1996.

58. Arain M, Campbell MJ, Cooper CL, et al. What is a pilot or feasibility study? A review of current practice and editorial policy. BMC Med Res Methodol 2010;10:67.

59. Braun V, Clarke V. Using thematic analysis in psychology. Qual Res Psychol 2006;3:77-101.
60. Smith BE, Hendrick P, Bateman M, et al. Study protocol: a mixed methods feasibility study for a loaded self-managed exercise programme for patellofemoral pain. Pilot Feasibility Stud 2018;4:24.

61. Fetters MD, Curry LA, Creswell JW. Achieving integration in mixed methods designs-principles and practices. Health Serv Res 2013;48(6 Pt 2):2134-56.

62. Evidence-based Review of Moderate to Severe Acquired Brain Injury (ERABI). https://www.abiebr.com (Accessed 3 Apr 2018). 